\title{
Android-Based Disease Monitoring
}

\author{
Woon Chee Kok ${ }^{1}$, Jane Labadin², 'Aisyah Mohammad, Kok Siong Wong, Yei Ling Chang, \\ \# Department of Computational Science and Mathematics \\ Faculty of Computer Science and Information Technology, Universiti Malaysia Sarawak. \\ 94300, Kota Samarahan, Sarawak, Malaysia. \\ 15020339@siswa.unimas.my \\ ${ }^{2}$ ljane@unimas.my
}

\begin{abstract}
Intensive worldwide effort is speeding up the developments in the establishment of a global surveillance network for combating pandemics of emerging infectious disease. However, there is no delivery platform available to send accurate and just-in-time disease information to reach out to the community. The public can only obtain information from the newspaper, radio or social media, which sometimes may not be an accurate information and can be classified as rumors. An android-based disease monitoring system has been implemented with a strong predictive model to help create awareness in our community. This monitoring system consists of 2 parts which are (i) Online Communicable Disease Monitoring System and (ii) Android-based monitoring application. In Malaysia, public health department utilizes Communicable Disease Control Information System to collect data and monitor diseases. However, the current system only collects the data from an authorized medical personnel but not utilizes the data to help the decision makers to access and monitor the spread of disease. The proposed system aimed to enhance the current system by providing better data visualization and incorporate forecasting component by using a mathematical model. Hand, Foot and Mouth Disease (HFMD) is used as a case study to demonstrate the system. Through the mobile application, the public user can be informed with real--time epidemic disease information from the proposed system.
\end{abstract}

Keywords - android application; disease monitoring system; Hand, Foot and Mouth disease; prediction model

\section{INTRODUCTION}

The global surveillance network is growing fast under an intensive worldwide effort. Surveillance is an ongoing systematic collection, analysis and interpretation of outcomespecific data for use in planning, implementing evaluating public health policies and practices. There are two main functions served by a communicable disease monitoring system (i) early alert and warning of potential threats to public health and (ii) programmed monitoring functions, which may be disease-specific or multi-disease in nature.

Hand, Foot and Mouth Disease (HFMD) become one of the most infectious diseases in Malaysia [1]. It mostly affects infants and young children under the age of five [2]. Symptoms normally begin with a fever, reduced appetite, sore throat, and a feeling of being unwell.
According to Datuk Seri Dr. S. Subramaniam, from January until April this year, a total of 13,497 cases were reported nationwide. This was an increase of $83 \%$ over the same period last year. The previous study mentioned that in avoiding close contact with the infected can reduce the risk of infection [3]. The Ministry of Health had raised awareness of HFMD especially in kindergartens and child care centers since the ministry expected the rise of HFMD in the year 2015. Prevention and control measures need to be taken in order to prevent a child from getting the HFMD.

In order to avoid close contact with the infected people, one needs to be aware of where the actual cases came from. Unfortunately, there is no delivery platform to make accurate and just-in-time information about the disease available for those uninfected community. Usually, public can only obtain information through mass media such as newspaper, radio or social media. However, sometimes such information does not come from trusted source and thus may not be accurate or worse just rumors. As a result, the public face difficulties in preventing HFMD at the earliest stage since the news and trusted information are disseminated to the public gradually. Therefore, in order to create awareness and help in reducing the infected cases of a disease, the authors implemented a disease monitoring system to integrate on a mobile application to disseminate real-time and reliable information to the community.

\section{MOTIVATIONS}

Infectious diseases are a leading cause of illness and death throughout the world. Today's infectious disease challenges are broader and more complex. People deal with infectious diseases with a passive approach which is an intervention that provides automatic protection without action from an individual. This is due to lack of accurate and just-in-time disease information from reliable authorized sources. If the public can obtain accurate and real-time disease information at their fingertips, this can create high awareness and the community can become proactive in preventing the disease. This can relieve the burden of the public health authorities and hospitals because unreliable disease information may lead in public plummeting at the hospital.

There is a nationwide collaboration that collect, manage, 